\title{
Human angiotensin-converting enzyme 2 transgenic mice infected with SARS-CoV-2 develop severe and fatal respiratory disease
}

\author{
Joseph W. Golden, ${ }^{1}$ Curtis R. Cline, ${ }^{2}$ Xiankun Zeng, ${ }^{2}$ Aura R. Garrison, ${ }^{1}$ Brian D. Carey, ${ }^{3}$ \\ Eric M. Mucker, ${ }^{1}$ Lauren E. White, ${ }^{4}$ Joshua D. Shamblin, ${ }^{4}$ Rebecca L. Brocato, ${ }^{1}$ Jun Liu, ${ }^{2}$ \\ April M. Babka, ${ }^{2}$ Hypaitia B. Rauch, ${ }^{3}$ Jeffrey M. Smith, ${ }^{1}$ Bradley S. Hollidge, ${ }^{1}$ Collin Fitzpatrick, \\ Catherine V. Badger, ${ }^{1}$ and Jay W. Hooper ${ }^{1}$ \\ 'Virology Division, ${ }^{2}$ Pathology, ${ }^{3}$ Diagnostic Systems Division, and ${ }^{4}$ Veterinary Medicine Division, United States Army \\ Medical Research Institute of Infectious Diseases (USAMRIID), Fort Detrick, Maryland, USA
}

\begin{abstract}
The emergence of SARS-CoV-2 has created an international health crisis, and small animal models mirroring SARS-CoV-2 human disease are essential for medical countermeasure (MCM) development. Mice are refractory to SARS-CoV-2 infection owing to low-affinity binding to the murine angiotensin-converting enzyme 2 (ACE2) protein. Here, we evaluated the pathogenesis of SARS-CoV-2 in male and female mice expressing the human ACE2 gene under the control of the keratin 18 promoter (K18). In contrast to nontransgenic mice, intranasal exposure of K18hACE2 animals to 2 different doses of SARS-CoV-2 resulted in acute disease, including weight loss, lung injury, brain infection, and lethality. Vasculitis was the most prominent finding in the lungs of infected mice. Transcriptomic analysis from lungs of infected animals showed increases in transcripts involved in lung injury and inflammatory cytokines. In the low-dose challenge groups, there was a survival advantage in the female mice, with $60 \%$ surviving infection, whereas all male mice succumbed to disease. Male mice that succumbed to disease had higher levels of inflammatory transcripts compared with female mice. To our knowledge, this is the first highly lethal murine infection model for SARS-CoV-2 and should be valuable for the study of SARS-CoV-2 pathogenesis and for the assessment of MCMs.
\end{abstract}

Conflict of interest: The authors have declared that no conflict of interest exists.

Copyright: ( $)$ 2020, Golden et al. This is an open access article published under the terms of the Creative Commons Attribution 4.0 International License.

Submitted: July 7, 2020 Accepted: August 20, 2020 Published: October 2, 2020

Reference information: /CI Insight. 2020;5(19):e142032. https://doi.org/10.1172/jci. insight.142032.

\section{Introduction}

SARS-CoV-2 is a betacoronavirus and the causative agent of COVID-19, a febrile respiratory human disease that initially emerged in China in late 2019 and subsequently spread quickly worldwide $(1,2)$ COVID-19 is primarily a respiratory disease with a wide spectrum of severity, ranging from a mild cough to the development of hypoxia, and in some cases resulting in a life-threatening acute respiratory distress syndrome requiring mechanical ventilation $(3,4)$. The most severe cases are generally skewed toward the aged population ( $>50$ years of age) and those with underlying health conditions such as hypertension or cardiovascular disorders $(5,6)$. SARS-CoV-2 human infections can also cause vasculature damage and coagulopathies, leading to infarction (7-9). Acute disease often presents with elevated levels of inflammatory cytokines, including IL-6, and these host molecules may play a role in the pathogenic process $(10,11)$ Additionally, approximately $30 \%$ of cases include signs of neurological disease such as headache, anosmia (loss of smell), ataxia, meningitis, seizures, and impaired consciousness (12-14). To date, global SARSCoV-2 cases have infected more than 8 million people and resulted in more than 400,000 deaths. Therefore, medical countermeasures (MCMs) are urgently needed to prevent this disease or to limit disease severity in a post exposure setting.

Similar to SARS-CoV (15), SARS-CoV-2 binds to target cells via an interaction between the $139-\mathrm{kDa}$ viral spike protein and the host angiotensin-converting enzyme 2 (ACE2) protein (16-19). An important factor in host tropism of the virus is this receptor interaction, and reduced affinity between these 2 molecules greatly impacts host susceptibility to infection. Both SARS-CoV and possibly SARS-CoV-2 bind to murine ACE2 (mACE), poorly compared with human ACE2 (hACE2) (20). Accordingly, mice are inherently refractory to infection by ACE2 using human coronaviruses (21-23). In these animals, infection by 
SARS-CoV (22) and SARS-CoV-2 (23) is rapidly controlled, although older mice are more permissive to lung replication; however, lung injury is limited and mortality is generally low. Moreover, mice lacking adaptive immunity owing to RAG deficiencies (no T cells or B cells) are protected against severe SARS-CoV disease, whereas mice genetically devoid of STAT-1, an important molecule involved in innate immunity, are sensitive to infection by SARS-CoV (24-26). However, disease in STAT-1 mice is protracted and not highly representative of human infections $(25,26)$.

In response to the need for small animal models to study SARS-CoV, several laboratories produced transgenic animals expressing the hACE2 gene under the control of various promoters, including the CMV, the HFH4 epithelial cell promoter, and the endogenous murine ACE2 promoter (27-31). Many of these animals develop severe disease upon infection by SARS-CoV, but severity largely depends on expression levels and tissue distribution of the hACE2 transgene. One transgenic mouse strain, termed "K18-hACE2," developed by Perlman et al., expresses the hACE2 molecule under the control of the keratin 18 promoter (K18) (30, 32). K18 limits expression to airway epithelial cells and colon and to a lesser extent kidney, liver, spleen, and small intestine. A minor level of hACE2 expression was also detected in the brain. These mice are susceptible to SARS-CoV strain Urbani and develop severe respiratory disease after intranasal exposure characterized by lung inflammation, serum cytokine and chemokine production, and high lethality (30). As with several mouse strains transgenic for hACE2, SARS-CoV infects the brains of K18-hACE2 mice $(30,32)$. CNS localization is speculated to play a major role in host mortality owing to neuronal cell death, particularly cell loss in the cardiorespiratory control center (32). It has recently been reported that some of these hACE2 transgenic mice are being used for SARS-CoV-2 research and that newer systems have been developed using CRISPR/Cas9 $(23,33,34)$. However, none were shown to produce a consistent lethal disease, and in models where lung injury occurred, it was most pronounced in aged animals $(23,34)$. Here, we evaluated susceptibility of K18-hACE2 mice to SARS-CoV-2. We found that mice developed severe disease that included respiratory distress with weight loss and mortality, as well as brain infection.

\section{Results}

SARS-CoV-2 produces severe and fatal infection of K18-hACE2 mice. Two groups of 9-week-old K18-hACE2 mice ( $n=14$ per group) were intranasally infected with $2 \times 10^{4}$ or $2 \times 10^{3}$ PFU of SARS-CoV-2. These groups were equally divided by sex ( $n=7$ per group/sex). On day 3, 2 mice per group were euthanized to assess disease severity. The remaining 5 mice per group were monitored up to 28 days. We also infected C57BL/6, $\mathrm{BALB} / \mathrm{c}$, and RAG2-deficient mice with a challenge dose of $2 \times 10^{4} \mathrm{PFU}$. On day 4 , all groups of infected K18-hACE2 mice began to lose weight, which was more pronounced in the female mice compared with the male mice in either challenge dose group (Figure 1A and Supplemental Figure 1A; supplemental material available online with this article; https://doi.org/10.1172/jci.insight.142032DS1). Nontransgenic mice did not lose any weight, and no animal succumbed to disease. Starting on day 5, several K18-hACE2 animals began to show signs of respiratory disease, including labored breathing and conjunctivitis. On day 5 through day 7 , most mice $(15 / 20$ mice $)$ began to meet euthanasia criteria $(n=13)$ or died $(n=2)$. An additional animal in the low-dose male group succumbed to disease on day 11, after a period of weight loss. The highest mean weight loss was in the female groups ( $>20 \%$ ), although male mice lost more than $12 \%$ of their weight (Supplemental Figure 1, A and B). Of the K18-hACE2 mice in the high-dose challenge groups, all female mice and $80 \%$ of the male mice succumbed to disease. Most female mice survived in the low-dose group, with $40 \%$ mortality, but all male mice succumbed to disease. The difference in survival between low-dose male and female mice was significant (log-rank; $P=0.040$ ), as was mortality between the high- and lowdose female groups (log-rank; $P=0.037$ ). There was no statistical significance in survival between the other groups. Lung homogenates taken on day 3 showed high levels of virus in K18-hACE2 mice, in contrast to C57BL/6 or RAG2-deficient mice, which had low levels of virus (Figure 1, B and C). Virus RNA levels in lung, liver, and spleen were nearly identical between all the infected K18-hACE2 groups and remained high in most of the euthanized mice, apart from the mouse that died on day 11 (Figure 1, B and C, and Supplemental Figure 1C). That animal had markedly lower levels of detectable genomic RNA (Figure 1C). Compared with nontransgenic mice and uninfected K18-hACE2 mice, infected K18-hACE2 mice had comparatively higher serum levels of TNF- $\alpha$, IL- 6 , and IL-10 as well as the monocyte chemoattractants MCP-1 (CCL2) and MCP-3 (CCL7) (Figure 1D). Cytokine levels observed in mice that succumbed to disease were generally higher compared with those sampled on day 3. Overall, these findings indicated that SARS-CoV-2 causes severe disease in K18-hACE2 mice following intranasal exposure. 
A

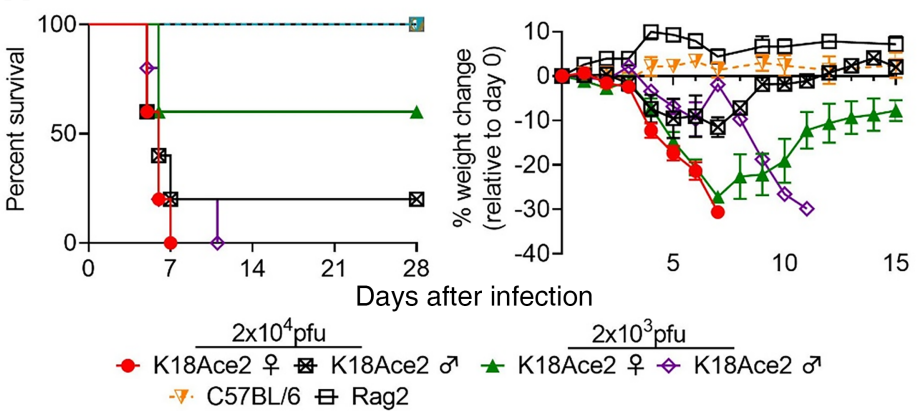

B

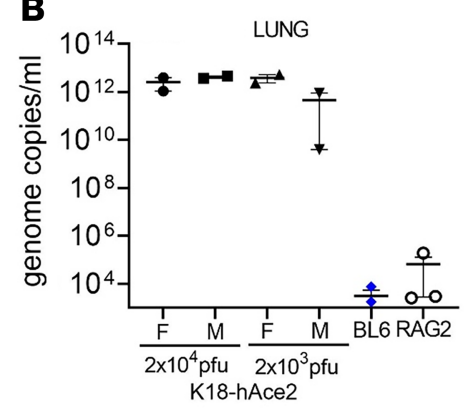

C

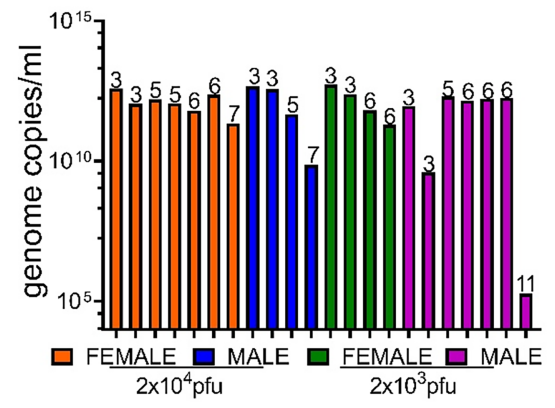

D
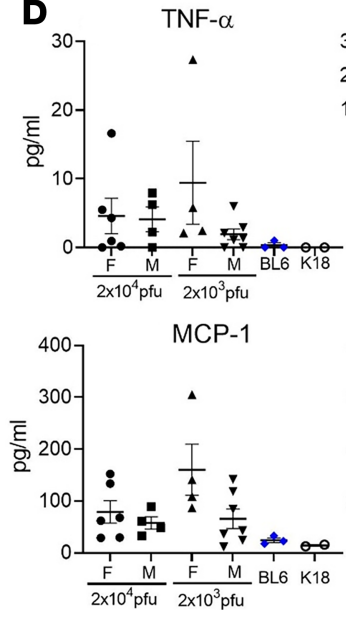
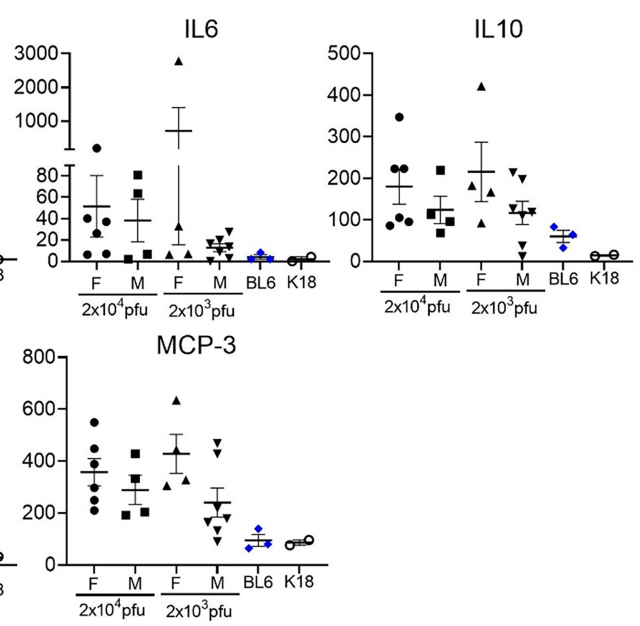

Figure 1. SARS-CoV-2 infection in K18-hACE2 transgenic mice. (A) Male and female K18-hACE2 transgenic mice (day $0-3, n=7 /$ group; day $3+, n=5 /$ group) were infected with $2 \times 10^{4} \mathrm{PFU}$ or $2 \times 10^{3} \mathrm{PFU}$ of SARS-CoV-2 by the IN route. C57BL/6 and RAC2 KO mice (day $0-3, n=8 /$ group; day $3+, n=5 / \mathrm{group}$ ) were infected with $2 \times 10^{4} \mathrm{PFU}$ by the IN route. Survival and weight loss $( \pm$ SEM) were monitored and plotted using Prism software. (B) Titers in lung ( $n=2$ mice/ group) were examined on day 3 by qRT-PCR. Mean titers \pm SEM of the genome molecules of viral RNA/mL were graphed. (C) Titers in lungs of individual K18-hACE2 mice. Numbers above bars denote day of death. Colors represent the 4 groups. (D) Monocyte chemoattractants and inflammatory cytokines were measured from the serum of SARS-CoV-3-infected mice on day 3 or at the time of euthanasia using a multiplex system. Mice from each group are aggregated from samples taken on day 3 (blue symbols) and when mice were euthanized (black symbols).

Lungs of K18-hACE2 mice exposed to SARS-CoV-2 show signs of acute disease. Lungs were collected from K18hACE2 mice on day 3 or at the time of euthanasia owing to disease severity. In K18-hACE2 mice, viral RNA was detected by ISH in all mice on day 3 and in most mice succumbing to disease on days 5-7; however, these levels diminished as disease progressed (Figure 2A, Supplemental Figure 2, and Supplemental Table 1). Additionally, ISH labeling was patchy (Supplemental Figure 2) and most severe at the day 3 time point. ISH labeling was present in both inflamed and normal-appearing alveolar septa. Positive ISH labeling for SARS-CoV-2 was identified multifocally in alveolar septa in the lung, suggesting infection of pneumocytes and macrophages. This was confirmed by the detection of SARS-CoV-2 protein in E-cadherin ${ }^{+}$cells (pneumocytes) and $\mathrm{CD} 8^{+}$(alveolar and infiltrating macrophages) using immunofluorescence assay (IFA) (Figure 2, B and C).

In comparison with the normal lung architecture in uninfected control animals, infected mice necropsied on day 3 , and those succumbing to disease on days 5-11 had varying levels of lung injury, including area of lung consolidation characterized by inflammation and expansion of alveolar septa with fibrin, edema, and mononuclear leukocytes, as well as infiltration of vessel walls by numerous mononuclear leukocytes (Figure 3, A-D, Supplemental Figure 4, and Supplemental Table 1). Type II pneumocyte hyperplasia was identified in less than one-half of infected animals. This lesion had a relatively patchy distribution except in the most severely affected animals in which it was more abundant. In areas of septal inflammation, exudation of fibrin and edema into alveolar lumina from damaged septal capillaries was observed in most animals. Vasculitis was the most common finding and was present in approximately $95 \%$ of all mice (Figure 3E). The lesion encompassed small and intermediate caliber vessels and was often characterized by near circumferential infiltration of the vessel wall by numerous mononuclear inflammatory cells. This lesion also contained small amounts of fibrin and occasional necrotic debris, 
A
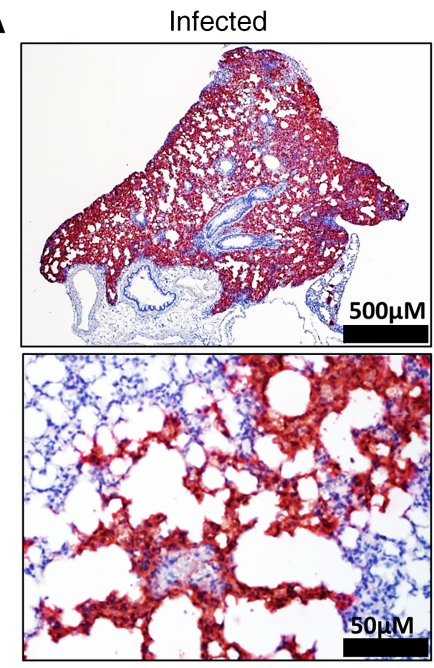

$\mathbf{B}$

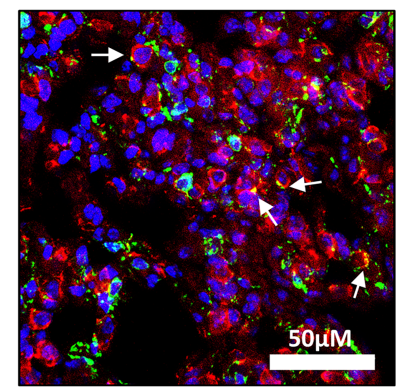

Spike E-cadherin
C

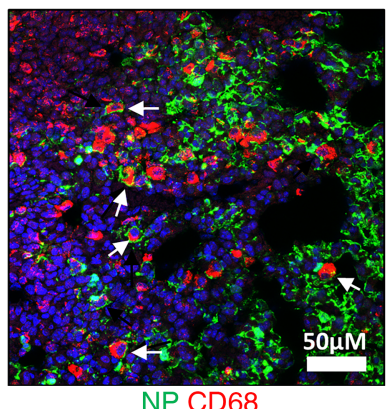

Figure 2. Infection of SARS-CoV-2 in the lung of K18-hACE2 transgenic mice. (A) Representative ISH images showing the presence of SARS-CoV-2 RNA (red) in the lungs of infected mice at low and high magnification or uninfected mice. Cells were counterstained with hematoxylin (blue). (B) Costaining for viral spike protein (green) and E-cadherin (red) in infected lung tissues using IFA. Arrows point to double-positive cells. Nuclei are stained with DAPI (blue). (C) Costaining of viral nucleoprotein and the macrophage marker CD68 (red) in infected lungs using IFA. Arrows denote double-positive cells. Nuclei are stained with DAPI (blue).

affecting all tunics and obscuring the vessel wall architecture. However, the endothelial cells surrounding lung vasculature were largely free of viral RNA (Figure 3E). In 1 low-dose male mouse that died on day 11, evidence of numerous fibrin thrombi was identified in the small and intermediate vessels (Figure 3B), suggestive of a hypercoagulable state within the lung. This same animal had marginally detectable virus in the lung (Figure 1C), and fibrin thrombi were not identified in other organs, including liver and kidney. Infected K18-hACE2 mice had elevated numbers of TUNEL ${ }^{+}$cells, suggesting increased cell death (Figure $3 \mathrm{~F}$ ). We also detected increased Ki-67 expression, a marker for cellular proliferation, most likely by proliferating pneumocytes and potentially by replicating alveolar macrophages (Figure $3 \mathrm{G}$ ). Neutrophilia was detected by $\mathrm{H} \& \mathrm{E}$ staining, consistent with an increase in myeloperoxidase $\left(\mathrm{MPO}^{+}\right)$cells (neutrophils, basophils, and eosinophils) detected by IFA (Supplemental Figure 3A). However, the $\mathrm{MPO}^{+}$cells were devoid of viral antigen (Supplemental Figure 3A). There was also an increase in the presence of CD68 macrophages (Supplemental Figure 3B) and a pronounced increase in $\mathrm{CD}^{+} 5^{+}$and $\mathrm{CD}^{+}$cells in infected lungs, indicative of infiltrating leukocytes, including $\mathrm{T}$ cells, which is consistent with histologic findings (Supplemental Figure 3C). These data indicate that K18-hACE2 mice develop a pronounced lung injury upon exposure to SARS-CoV-2.

Transcriptional profiles of host immunological and inflammatory genes in lung homogenates from SARS-CoV-2-infected K18-hACE2 and C57BL/6 mice were examined by NanoString-based gene barcoding on day 3 (K18-hACE2 and C57BL/6) or in K18-hACE2 mice at the time of euthanasia. A total of 478 transcripts increased in K18-hACE2 mice and at 430 decreased at a $\log _{2}$ fold cutoff of 1 and a $P$ value less than 0.05. Many of the increased transcripts in the K18-hACE2 mice were inflammatory genes, including IL-6, IFN- $\gamma$ receptor-1, and chemokines (CCL2, CCL5, CCL9, and CXCL10) (Figure 4A). 


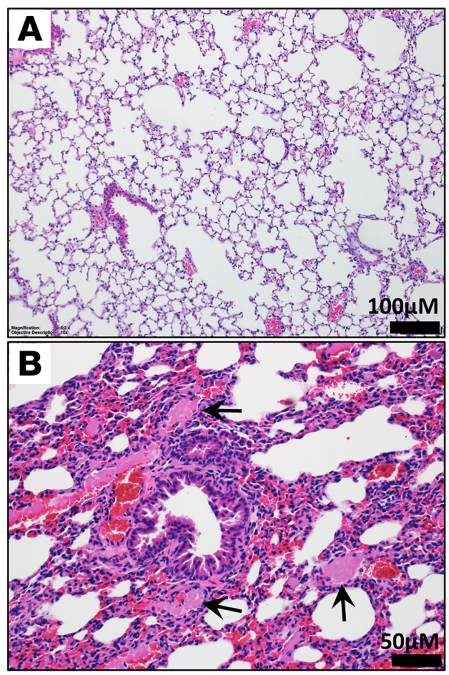

E

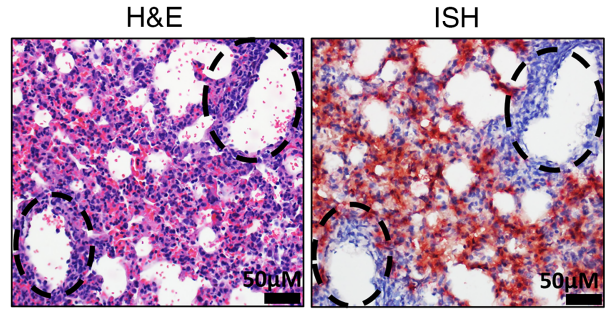

$\mathbf{F}$
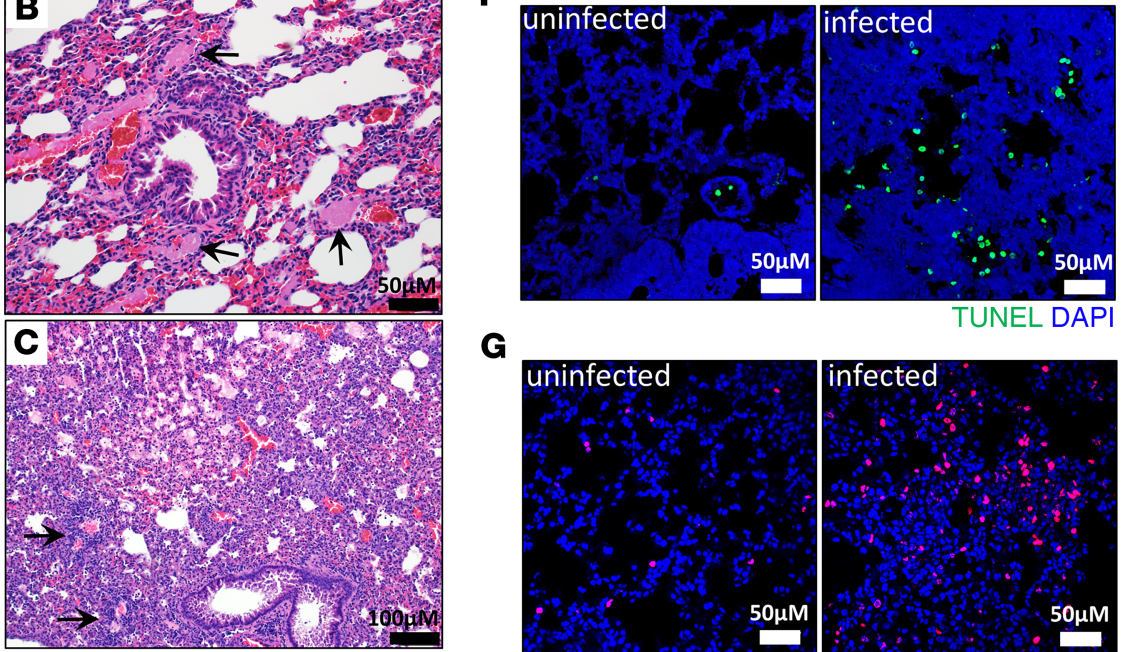

G
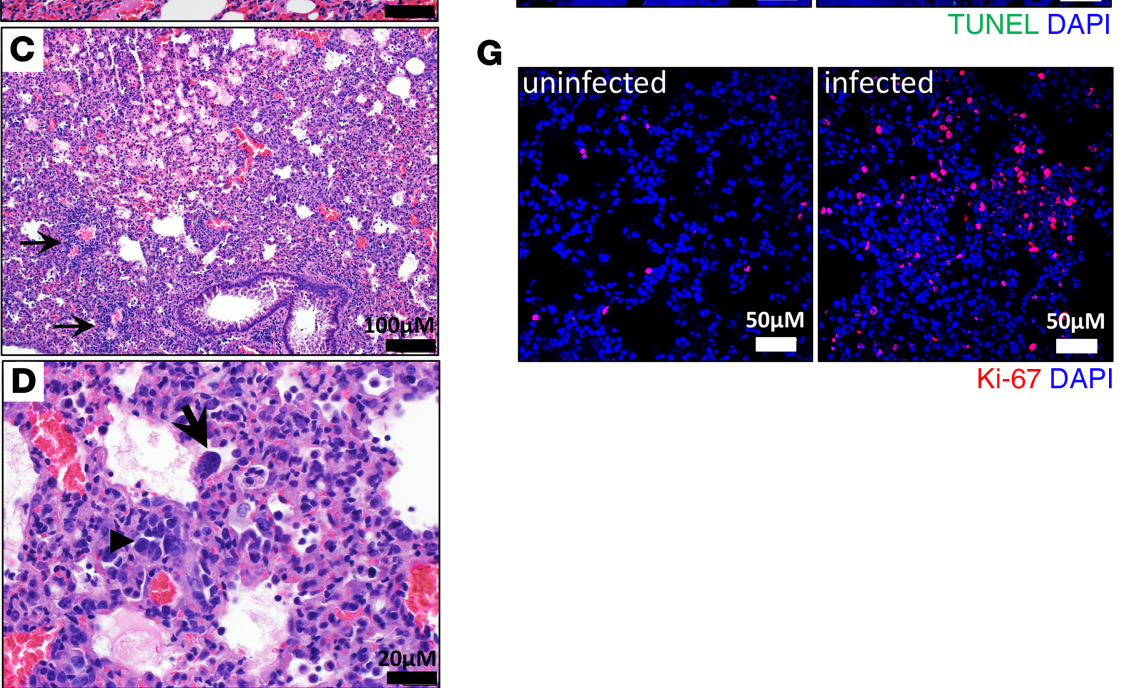

Figure 3. SARS-CoV-2 infection causes respiratory damage in K18-hACE2 mice. (A-D) Representative H\&E staining of lungs in infected C57BL/6 mice (A) or K18-hACE2-infected mice (B-D). Numerous fibrin thrombi (black arrows) filling the lumen of small-to-intermediate size vessels (B) adjacent to a normal bronchus and surrounded by minimally inflamed, congested, and collapsed alveolar septa. (C) Extensive area of lung consolidation with inflammation and expansion of alveolar septa, exudation of fibrin and edema into alveolar lumina, and infiltration of vessel walls and perivascular area by numerous mononuclear inflammatory cells (arrows), disrupting/obscuring vessel architecture (vasculitis). (D) Extensive area of consolidated lung showing type II pneumocyte hyperplasia (arrowhead) and rare multinucleate cells (black arrow). (E) H\&E and ISH staining of infected mouse lung showing vasculitis with absence of viral RNA in the affected vessel walls; note there is viral RNA (red) in the adjacent alveolar septa. Highlighted vessels (broken black circles). (F) TUNEL staining of infected and uninfected K18-hACE2 mouse lungs. TUNEL (green) was performed as indicted in Methods. Cell nuclei stained with DAPI (blue). (C) Ki-67 staining (red) in infected and uninfected K18-hACE2 mouse lungs. Nuclei stained with DAPI (blue).

Type I and type II IFN transcripts IFNA1, IFNB1, and IFNG, along with the cytokines IL-9 and IL-2, decreased. Consistent with the increased presence of $\mathrm{CD} 68^{+}$macrophages, $\mathrm{CD} 68$ transcripts also substantially increased in K18-hACE2 mice. Viral sensing pathways were elevated in infected mice with severe disease, indicated by high transcript levels of Irf1, RIG-I (Ddx58), and MDA-5 (Ifih1). Among the highest activated transcripts in the K18-hACE2 mice were genes involved in lung injury and hypoxia, including sphingosine-1-phosphate lyase 1 (Sgpl1), resistin-like molecule- $\alpha$ (Retnl $\alpha$, FIZZ, and HIMF), stromal cell-derived factor 1 (SDF1/CXCL12), and HIF1- $\alpha$. No major differences were observed between the challenge doses. A total of 13 genes increased and 21 genes decreased between the male and female mice (Figure 4B). Male mice had higher levels of inflammatory cytokine transcripts, including CXCL2, IL6, IL1R2, and CXCL1. Transcripts expressed more highly in female animals included fatty acid synthase and histone demethylase UTY. Collectively, these findings indicate that K18-hACE2 mice develop transcriptomic signatures of lung injury 
after exposure to SARS-CoV-2 with increased expression of inflammatory genes, inflammatory gene transcripts, and markers of lung injury and hypoxia.

SARS-CoV-2 is present in the nasal cavity and eyes of K18-hACE2 mice. SARS-CoV-2 RNA was observed in the nasal turbinates in most mice and rarely within the eyes of infected mice (Figure 5A, Supplemental Figure 5, and Supplemental Table 1). Viral RNA in the eye was localized to the retina, suggesting viral infection of neurons in the inner nuclear layer and ganglion cell layer (Supplemental Figure 5). Despite infection, evidence of inflammation or other damage in the retina or elsewhere in the eye was not present. Viral RNA and spike protein were also detected to some degree in the nasal turbinate epithelium (predominantly olfactory epithelium) as early as day 3 after infection (Figure 5B), as indicated by costaining with cytokeratin (Figure 5C). Pathology was minimal, predominantly isolated to few areas of olfactory epithelium atrophy, with degeneration or erosion present in the epithelium lining the dorsal and lateral nasal meatuses (Figure 5D). Some cellular sloughing was also detected, and these sloughed cells contained viral RNA (Supplemental Figure 5). In mice succumbing to disease on days 5-7, virus was present in the olfactory bulb, and most animals showed asymmetrical staining, with one bulb more positive than the other. Viral RNA was detected throughout the olfactory bulb, including in the olfactory nerve layer, glomerular layer, external plexiform layer, and mitral cell layer of olfactory bulbs, in most of the animals. Viral protein colocalized with the neuronal marker NeuN, suggesting virus was present within neurons in the olfactory bulb (Supplemental Figure 6). Virus was not detected in the olfactory bulb of animals taken on day 3 , suggesting that virus trafficked to this region on day 4 or 5 . These data indicated that SARS-CoV-2 infects cells within the nasal turbinates, eyes, and olfactory system and that infection was observed in epithelial cells and neurons.

$S A R S$-CoV-2 infects the brain of K18-hACE2 mice. Brain infection was not observed in most of the animals examined on day 3 but was prevalent in mice necropsied on days 5-11 (Supplemental Table 1). Evidence of SARS-CoV-2 was found throughout the brain, including strong but variably diffuse signal in regions of the thalamus, hypothalamus, amygdala, cerebral cortex, medulla, pons, and midbrain (Figure 6, A-C, and Supplemental Table 1). A similar intense but less diffuse signal was present within the hippocampus. $\mathrm{ISH}^{+}$cells included neurons of thalamic nuclei. In contrast, cells within the vessel walls and perivascular spaces infiltrated by mononuclear inflammatory cells were negative for viral genomic RNA (Figure 6C). Histopathological changes were detected in the brains of several infected K18-hACE2 mice euthanized on days 5-11 but not in most mice euthanized on day 3 (Figure 6, D-G; Supplemental Table 1; and Supplemental Figure 7). In the thalamus and hypothalamus, vasculitis was the most common lesion, characterized by endothelial hypertrophy and increases in mononuclear leukocytes within the vessel wall and/or filling the perivascular space. Small amounts of necrotic debris were also identified. In most of these cases, the vascular lesion was characterized by the presence of increased numbers of microglia within the adjacent neuropil. Occlusive fibrin thrombi were also detected within the thalamus in a few mice. Meningitis was observed in a subset of animals. It was associated with infiltration of mononuclear leukocytes (most lymphocytes) and most prominent adjacent to vessels. In the mouse that died late on day 11, with massive pulmonary clotting, the rostral cerebral cortex brain lesions included small-to-intermediate size vessel walls multifocally expanded by mononuclear inflammatory cells and perivascular hemorrhage extending into the adjacent neuropil (Figure 6E). Increased numbers of microglia were readily detected on H\&E-stained sections, expanding outward from the perivascular neuropil. An increased number of microglia was found in the neuropil surrounding affected vessels. Additionally, brains also showed signs of neuroinflammation, indicated by increased staining of Iba-1 and GFAP, indicating microgliosis and astrocytosis, respectively (Figure $6 \mathrm{H})$. Necrosis was identified in at least 5 animals and was most prominent within the periventricular region of the hypothalamus as well as in the amygdala. The lesion was characterized by moderate numbers of shrunken, angular cells with hypereosinophilic cytoplasm and pyknotic nuclei and surrounded by a clear halo (Supplemental Figure 7). The morphology and location of individual cells was suggestive of neuronal necrosis, but further investigation will be required to confirm this finding. Viral spike protein was detected in $\mathrm{NeuN}^{+}$cells, indicating viral infection of neurons (Figure 6I). Viral antigen was absent in $\mathrm{GFAP}^{+}$cells, suggesting the virus does not productively infect astrocytes. These data indicate that similar to SARS-CoV, SARS-CoV-2 also targets the brain of K18-hACE2 mice, causing brain injury. As indicated by duplex ISH labeling of brain, neurons are positive for both hACE2 transgene expression and viral genomic RNA (Supplemental Figure 8). No animal showed outward signs of neurologic deficit, such as hind limb paralysis, head tilting, or tremors. 
A

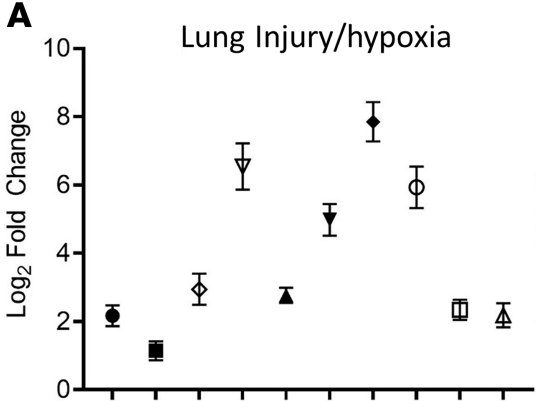

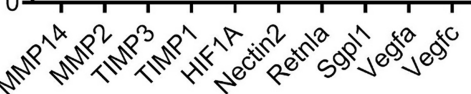
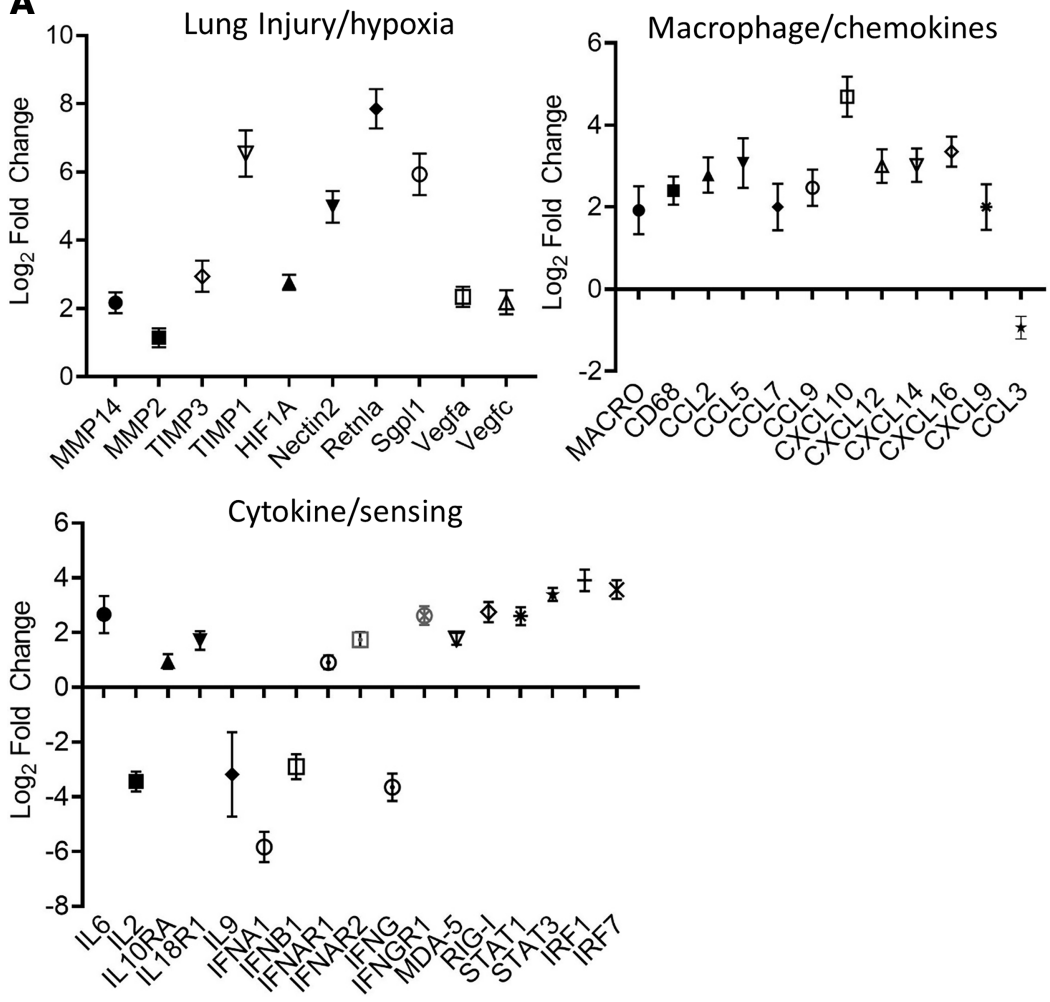

B

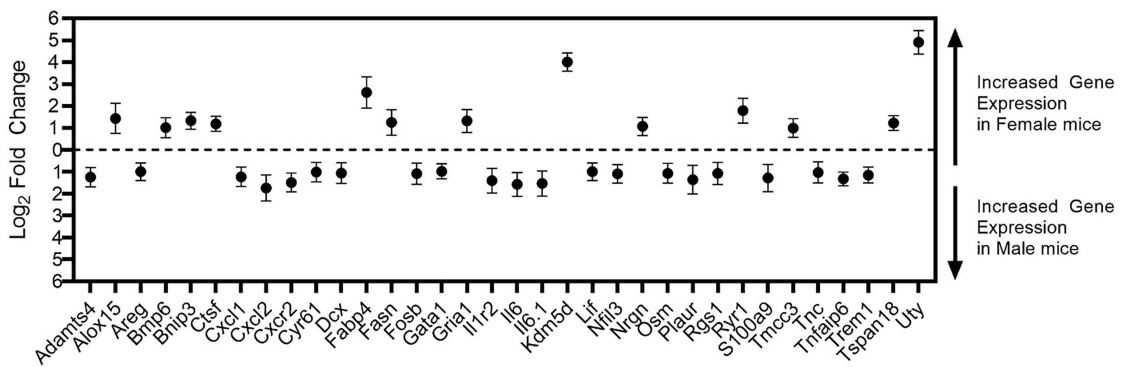

Figure 4. Transcriptional activation in SARS-CoV-2-infected lungs. Transcriptional activation in lung homogenates from infected K18-hACE2 (male and female) and C57BL/6 (female) mice were examined by NanoString. (A) Log fold $_{2}$ changes in gene expression levels of selected genes categorized by group vs. infected C57BL/6 mice were graphed with SD. All graphed transcripts had a $P$ value of less than 0.05. (B) Differential gene expression $\left(\log _{2}\right.$ changes) between infected male and female K18-hACE2 mice. All graphed transcripts had a $P$ value of less than 0.05 .

\section{Discussion}

Other murine infection models for SARS-CoV-2 involving transgene expression of the human ACE2 protein have previously been reported $(23,33,34)$. However, in these models, SARS-CoV-2 only produces a transient weight loss with some lung injury, but the animals generally recover. Additionally, several of these models required mice aged more than 30 weeks for the most severe disease to occur, diminishing the practicality of these systems given the urgent need for MCMs $(23,34)$. One system tested SARS-CoV-2 infection in mice in which hACE2 was expressed under the control of the HFH4 promoter (33). Infection in these mice was only approximately $40 \%$ lethal ( $2 / 5$ mice), and lung injury (assessed by plethysmography) and weight loss were absent. Lethality in this system was exclusive to animals in which virus was detected in the brain. Other recently reported SARS-CoV-2 murine models involved transduction of mouse lungs, with a replication-incompetent adenovirus, or an adeno-associated virus, encoding the hACE2 gene $(35,36)$. Transduced lung cells expressing hACE2 supported SARS-CoV-2 replication, and lung pathology ensued along with weight loss. However, disease was generally mild with no lethality. Blockade of the type I IFN system using pharmacological intervention was needed to produce the most severe disease (35). 
A
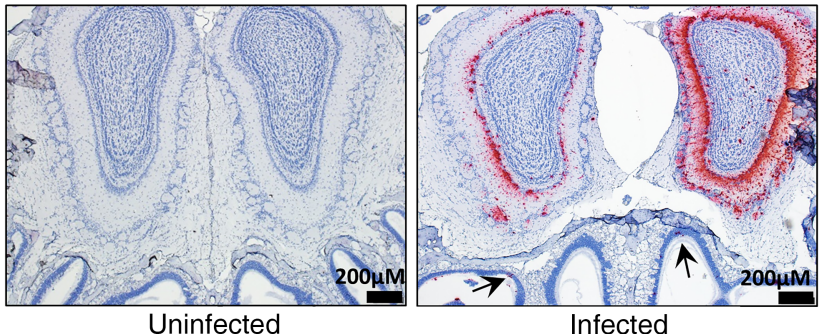

B

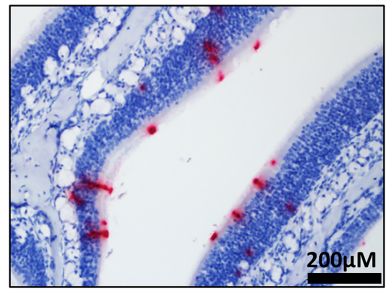

C

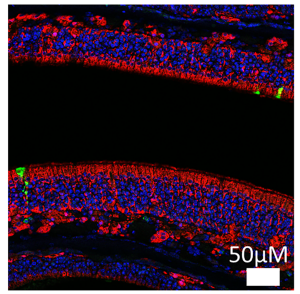

Spike Pan-cytokeratin

D

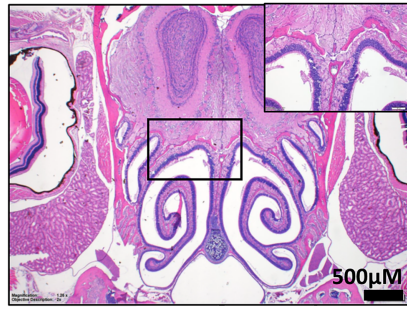

Uninfected

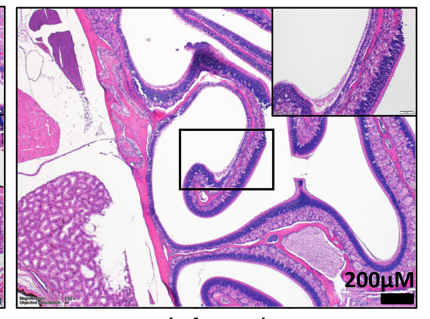

Infected

Figure 5. Infection of the nasal cavity and olfactory bulb in K18-hACE2 mice. (A) ISH labeling for SARS-CoV-2 RNA in a coronal section of the head, including the caudal aspect of the nasal cavity and olfactory bulb. Within the olfactory bulb, a strong positive signal is present in the glomerular layer, external plexiform layer, mitral cell layer, and internal plexiform layer, with multifocal positivity in the granular cell layer in the olfactory bulb hemisphere at right. Low numbers of cells within the olfactory epithelium lining the dorsal nasal meatus have a positive ISH signal (arrows). Cells were counterstained with hematoxylin (blue). (B) ISH labeling for SARS-CoV-2 RNA in the olfactory epithelium. Cells were counterstained with hematoxylin (blue). (C) IFA of olfactory epithelium stained for SARS-CoV-2 spike (green) and Pan-cytokeratin (red). Nuclei were stained with DAPI (blue). (D) Representative H\&E staining of the nasal cavity, including olfactory epithelium and olfactory bulb from uninfected or infected mice. In the infected mouse, there is a focal area of olfactory epithelium atrophy on a nasal turbinate located in the lateral nasal meatus. Inset, indicated area of detail under increased magnification.

Although these less severe disease murine models will be beneficial in the evaluation of vaccines and other anti-infectives, systems faithfully reproducing the major elements of severe disease observed in humans will be essential for identifying the most promising MCMs aimed at abating acute SARS-CoV-2 human infections. Additionally, the K18-hACE2 mouse system may provide a platform for the rigorous screening of candidate drugs before their evaluation in other animal models. The K18-hACE2 mice lost considerable weight ( $>12 \%$ in males and $>20 \%$ in females), and lethality in the high dose exceeded $90 \%$. Acute lung injury was detected in all animals succumbing to disease, with vascular damage the most common lesion. Similar to findings with SARS-CoV, SARS-CoV-2 infected the brains of K18-hACE2 mice. Brain infection resulted in vasculitis and inflammation, with SARS-CoV-2 antigen detected in neurons. It is possible virus enters the brain via the olfactory bulb, as has been reported for SARS-CoV, although more studies will be required as virus may also have entered the brain via inflamed vessels. Whether mortality results directly from brain infection is not clear; however, this has been speculated as the major cause of mortality in SARS-CoV-infected mice (32). Infection in the brain was delayed by at least 4 days, as it was an uncommon finding in day 3 animals. Thus, during early infection, lung appears to be the primary target. The high er degree of disease severity observed in the K18-hACE2 mice versus other hACE2 transgenic mice is likely owing to the enhanced levels of the hACE2 transgene, as multiple copies of the molecule are expressed in 

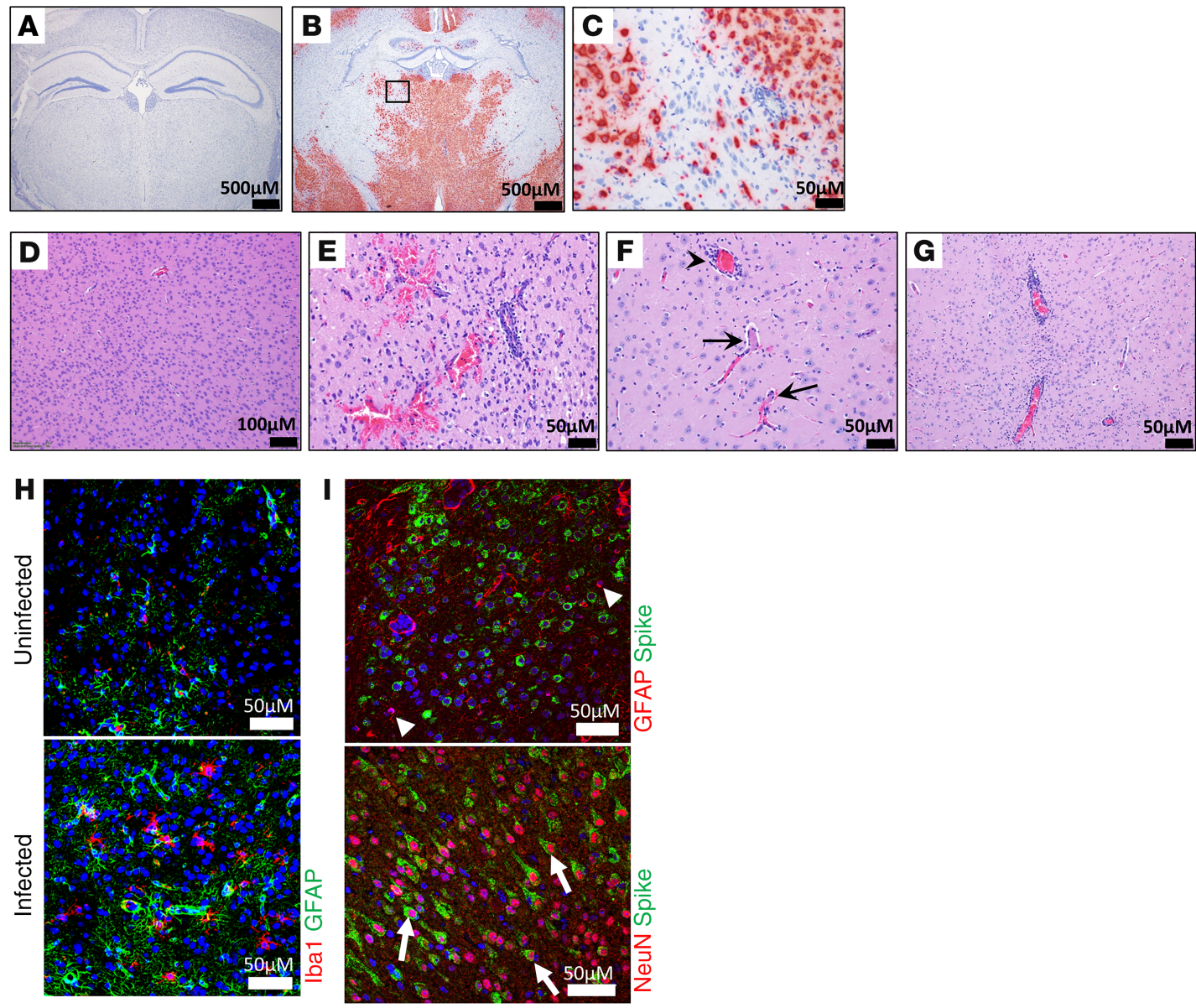

Figure 6. Neuropathogenesis of SARS-CoV-2 in K18-hACE2 transgenic and nontransgenic mice. ISH detection of for SARS-CoV-2 RNA in uninfected (A) and infected mice (B and $\mathbf{C}$ ) in a coronal section of brain demonstrating a strong positive signal within neurons of thalamic nuclei. The boxed area is shown at increased magnification (right) (C). Note the absence of a positive signal from the vessel at center right (C) where the vessel wall and perivascular space are infiltrated by mononuclear inflammatory cells. (D-G) Representative H\&E staining of uninfected (D) or infected (E-G) mice. Perivascular hemorrhage extending into the adjacent neuropil (E), in the region of the rostral cerebral cortex. Two small caliber vessels (F) in the thalamus with fibrin thrombi (arrows), with mild microgliosis and some perivascular hemorrhage (arrowhead). The walls of small-to-intermediate size vessels and perivascular spaces (G) are multifocally expanded/obscured by mononuclear inflammatory cells and increased numbers of glial cells. (H) Detection of Iba-1 and GFAP, markers for microgliosis and astrogliosis, respectively, in uninfected or infected brain sections using IFA. Nuclei were stained with DAPI (blue). (I) Costaining for astrocyte marker GFAP (red) or neuron marker NeuN (red) with SARS-CoV-2 spike protein (green). Spike protein was predominantly detected in NeuN ${ }^{+}$ neurons. Nuclei were stained with DAPI (blue).

murine cells, and it is well known that hACE2 levels modulate the disease severity of SARS-CoV (27-31). We have not yet evaluated the protective efficacy of MCMs in this model. However, it has been reported that antibodies protect against SARS-CoV, demonstrating the K18-hACE2 system is useful for evaluating countermeasures against ACE2-targeting human coronaviruses. Notably, in our study some animals at the lower dose survived infection despite significant weight loss ( $>20 \%$ in female mice), indicating the model is not hypersensitive to infection and that animals can recover. The K18-hACE2 animal model is commercially available and thus should provide an important platform to evaluate MCMs against SARS-CoV-2.

Infection of K18-hACE2 mice by SARS-CoV-2 produces a disease similar to that observed in acute human cases, with development of an acute lung injury associated with edema, production of inflammatory cytokines, and the accumulation of mononuclear cells in the lung. Impacted lungs had elevated levels of transcripts consistent with respiratory damage such as increased HIMF expression, which is involved in activation of lung endothelial cells in response to lung inflammation (37). We also found increased levels of Sgpl1, a molecule associated with lung injury (38) and known to increase in mechanically ventilated mice (39). Similar to transcriptional analysis of infected humans (40), we also observed decreases in expression of type I and type II IFN transcripts indicting SARS-CoV-2 can also suppress these IFN responses in mice, at least at the peak of disease. A prominent finding in infected K18-hACE2 mice system was vasculitis. 
Endotheliitis/vasculitis has been observed in human COVID-19 patients, and a role for the endothelium in acute disease is becoming more apparent $(7,8,41)$. Direct viral infection in human endothelial cells has also been reported (7). Notably, in mice virus was absent in these areas, suggesting vasculitis was attributed to host inflammatory processes. However, further study will be required to determine if vascular damage is incurred by direct or indirect viral effects. It has been speculated that during human acute disease, inflammatory cytokines (IL-6 in particular) are major drivers of tissue damage, and this is supported by data from humans showing IL-6 receptor targeting, with pharmacological antagonists (tocilizumab), can reduce morbidity (42). We found IL-6 transcripts, as well as chemokines, are elevated in mouse lungs. During human disease, pulmonary inflammation is associated with increases in lung granulocytes and an increase in macrophages $(4,43,44)$. Some have speculated that these macrophages play an important role in host injury (45). It is still unclear, however, if human macrophages are directly infected by SARS-CoV-2, although we found virus antigen in $\mathrm{CD}^{+} 8^{+}$macrophages and others report infection in murine $\mathrm{MAC}^{+}$macrophages (23). Further study will be required to determine the role macrophages play in SARS-CoV-2 lung injury and murine systems may be well suited. In addition to vascular issues, coagulopathies are a common finding in humans (9), with pulmonary embolism having been reported, along with clotting abnormalities leading to loss of limbs (46). At least some mice produced evidence of these clotting issues, with 1 mouse presenting with fibrin thrombi in the lungs.

During human infections, males have been reported to have more severe outcomes despite a similar infection rate (47). In our experiments, we observed a statistically significant difference in survival of female and male mice infected at the lower dose of virus, with $60 \%$ of females but no males surviving infection. Despite surviving, the female mice challenged with the low dose still lost a significant amount of weight $(>20 \%)$. Transcriptomic profiling in mouse lungs indicated female mice that succumbed to disease had modestly lower levels of IL-6, CXCL-2, and IL-1RA, suggesting a less intense inflammatory response. This study only involved a small number of animals, and more work will be required to determine if the K18-hACE2 system recapitulates this sex difference in disease severity.

The neuroinvasive aspects of COVID-19 are becoming more appreciated (12). SARS-CoV-2 causes neurological sequelae in at least one-third of human cases, including headache, confusion, loss of smell, meningitis, seizures, and ischemic and hemorrhagic stroke. The pathophysiology of human neurological injury induced by SARS-CoV-2 is ambiguous, and a direct role of the virus has not been established. Other coronaviruses are associated with neurological disease, including HEV67N in porcine and in humans SARS-CoV and MERS-CoV (48). SARS-CoV-2-infected K18-hACE2 mice showed evidence of neuroinflammation, neurovasculitis, meningitis, and hemorrhage, emulating many of the human findings. SARS-CoV has been shown to infect human and mouse brains, and data suggest it replicates in neurons. In the K18-hACE mice, neuronal cells in the olfactory bulb, retina, and brain were positive for SARS-CoV-2 viral RNA and antigen, suggesting these cells are supportive of a productive infection. In the K18-hACE2 mice, we suspect virus accesses the brain via infection of the olfactory bulb and then spreads to neurons and other regions of the brain via connective neuron axons (32). In humans, virus may gain access to the brain by infection of peripheral neurons, such as those in the olfactory bulb (48). Infection of these cells may help explain the loss of smell associated with some COVID-19 cases. However, the virus may also gain access via disruption of the blood-brain barrier, as these were inflamed in most animals and neurovasculitis has been found in humans (49). Overall, the K18-hACE2 system may help shed light on this poorly studied area of human disease.

In addition to the application of the K18-hACE2 model for the evaluation of MCMs, we conclude that this model may also be important in the study of SARS-CoV-2 pathogenesis. The identification of host factors driving the severe pathogenic processes in the lung, such as the role of macrophages and IL-6 in driving lung damage, may provide critical insight into the molecular and cellular determinates of COVID-19 pathogenesis. Additionally, as neurological sequelae during COVID-19 are an important component to severe disease, the K18-hACE2 model may provide a more defined understanding of SARS-CoV-2 interaction with the CNS.

\section{Methods}

Viruses and cells. A third passage of SARS-CoV-2 strain WA-1/2020 viral stock was obtained from the CDC and is from a human nonfatal case isolated in January 2020. A master challenge stock of virus was propagated by making 2 passages in Vero76 cells in MEM with Earle's Salts (Corning) supplemented with 1\% GlutaMAX (Thermo Fisher Scientific), 1\% NEAA (Thermo Fisher Scientific), and 10\% heat-inactivated FBS (Thermo Fisher Scientific). After 3 days, supernatant was collected and clarified by low-speed centrifugation. 
Virus (P5 from the founder stock of virus) was quantified by plaque assay and determined to be endotoxin free. All virus work was handled in BSL-3 containment at USAMRIID.

Mice. C57BL/6J (BL6), Rag2 KO mice and K18-hACE2 mice [B6.Cg-Tg(K18-hACE2)2Prlmn/J] (6-8 weeks old) were purchased from the Jackson Laboratory. Mice under isoflurane anesthesia were challenged intranasally with the indicated dose of SARS-CoV-2 WA-1/2020 strain diluted in a total volume of $50 \mu \mathrm{L}$ (25 $\mu \mathrm{L}$ per nare) of PBS (Gibco). Mice were uniquely identified using ear tags (Stoelting Co.) and provided an acclimation period of at least 1 week before commencement of the experiment. Mice were supplied nutrient gel when weights began to decrease.

$R T$ - $q P C R$. Organ tissue was homogenized in $750 \mu \mathrm{L}$ Trizol reagent using a Tissuelyser II (spleen and liver) (QIAGEN) or gentleMACS dissociator system on the RNA setting (lung). RNA was extracted from Trizol per modifications to the manufacturer's protocol. Briefly, following the addition of chloroform and transfer of the aqueous layer, equal amounts of $70 \%$ ethanol (Decon Laboratories, Inc.) was added to the aqueous layer, then the nucleic acid was purified using the RNeasy Mini Kit (QIAGEN) according to the manufacturer's instructions. A NanoDrop 8000 was used to determine RNA concentration, which was then raised to $100 \mathrm{ng} / \mu \mathrm{L}$ in UltraPure distilled water. Samples were run in duplicate, $500 \mathrm{ng}$ per sample, on a Bio-Rad CFX thermal cycler using TaqPath 1-step real-time (RT-qPCR) master mix according to the CDC's recommended protocol of $25^{\circ} \mathrm{C}$ for 2 minutes, $50^{\circ} \mathrm{C}$ for 15 minutes, $95^{\circ} \mathrm{C}$ for 2 minutes, followed by 45 cycles of $95^{\circ} \mathrm{C}$ for 3 seconds and $55^{\circ} \mathrm{C}$ for 30 seconds. The forward and reverse primer and probe sequences are 2019-nCoV_N2-F, 5'-TTACAAACATTGGCCGCAAA-3', 2019-nCoV_N2-R, 5'-GCGCGACATTCCGAAGAA-3', and 2019-nCoV_N2-P, 5'-ACAATTTCCCCCAGCGCTTCAG-3'. The limit of detection for this assay is $1 \times 10^{4}$ copies. A synthetic RNA containing the RT-PCR assay target sequence was acquired from Biosynthesis, Inc. The approximate target copy number was determined using the nucleic acid sequence and the molecular weight of the synthetic RNA, and a stock at a known target copy number per milliliter was generated by resuspending the RNA in TE buffer. A standard curve was generated by serially diluting the stock standard curve in DNase/RNase-free water (Invitrogen) and running the curve concurrently with the tested samples.

Histology. Necropsy was performed on the indicated organs. Tissues were immersed in $10 \%$ neutral buffered formalin for 14 days. Tissues were then trimmed and processed according to standard protocols (50). Histology sections were cut at 5-6 $\mu \mathrm{m}$ on a rotary microtome, mounted onto glass slides, and stained with H\&E. Tissue examination was performed by a board-certified veterinary pathologist.

Cytokine and chemokine analysis. Serum cytokine and chemokine analyses were performed using a magnetic bead-based plex mouse panel (Thermo Fisher Scientific) targeting the indicated molecules. A total of $25 \mu \mathrm{L}$ serum per mouse per time point was used. Plates were analyzed on a MAGPIX system (MilliporeSigma) and quantitated against standard curves using MILLIPLEX analyst software.

In situ hybridization. To detect SARS-CoV-2 genomic RNA in FFPE tissues, ISH was performed using the RNAscope 2.5 HD RED kit (Advanced Cell Diagnostics) as described previously (51). Briefly, $40 \mathrm{ZZ} \mathrm{ISH} \mathrm{probes}$ targeting SARS-CoV-2 genomic RNA fragment 21571-25392 (GenBank LC528233.1) were designed and synthesized by Advanced Cell Diagnostics (854841). Tissue sections were deparaffinized with xylene, underwent a series of ethanol washes and peroxidase blocking, and were then heated in kit-provided antigen retrieval buffer and digested by kit-provided proteinase. Sections were exposed to ISH target probe pairs and incubated at $40^{\circ} \mathrm{C}$ in a hybridization oven for 2 hours. After rinsing, ISH signal was amplified using kit-provided preamplifier and amplifier conjugated to alkaline phosphatase and incubated with a Fast Red substrate solution for 10 minutes at room temperature. Sections were then stained with hematoxylin, air-dried, and coverslipped.

Immunofluorescence. FFPE tissue sections were deparaffinized using xylene and a series of ethanol washes. After $0.1 \%$ Sudan black B (MilliporeSigma) treatment to eliminate the autofluorescence background, the sections were heated in Tris-EDTA buffer (10 mM Tris base, $1 \mathrm{mM}$ EDTA solution, $0.05 \%$ Tween 20, $\mathrm{pH}$ 9.0) for 15 minutes to reverse formaldehyde cross-links. After rinses with PBS (pH 7.4), the sections were blocked with PBT (PBS $+0.1 \%$ Tween 20 ) that contained $5 \%$ normal goat serum overnight at $4^{\circ} \mathrm{C}$. Then, the sections were incubated with primary antibodies: rabbit polyclonal anti-SARS-CoV Spike at a dilution of 1:200 (40150-T62-COV2, Sino Biological), mouse monoclonal anti-SARS-CoV NP at a dilution of 1:200 (40143-MM05, Sino Biological), mouse monoclonal anti-pan cytokeratin at a dilution of 1:100 (sc-8018, Santa Cruz Biotechnology), mouse monoclonal anti-e-cadherin at a dilution of 1:100 (334000, Thermo Fisher Scientific), rabbit polyclonal anti-MPO at a dilution of 1:200 (A039829-2, Dako Agilent Pathology Solutions), rabbit polyclonal anti-CD3 antibody at a dilution of 1:200 (A045229-2, Dako 
Agilent Pathology Solutions), rat monoclonal anti-CD45 antibody at a dilution of 1:100 (05-1416, MilliporeSigma), rabbit polyclonal anti-CD68 at a dilution of 1:200 (ab125212, Abcam), mouse monoclonal anti-NeuN at a dilution of 1:200 (MAB377, MilliporeSigma), and/or chicken polyclonal anti-GFAP at a dilution of 1:200 (ab4674, Abcam) for 2 hours at room temperature. After rinses with PBT, the sections were incubated with secondary goat anti-rabbit or anti-chicken Alexa Fluor 488 at a dilution of 1:500 (Thermo Fisher Scientific) and goat anti-mouse or anti-rat Alexa Fluor 568 at a dilution of 1:500 (Thermo Fisher Scientific) antibodies, for 1 hour at room temperature. Sections were coverslipped using the Vectashield mounting medium with DAPI (Vector Laboratories). Images were captured on a Zeiss LSM 880 confocal system and processed using ImageJ software (NIH).

TUNEL staining. FFPE lung tissue sections were deparaffinized in Xyless II buffer (LabChem) 2 times for 10 minutes each and rehydrated using an ethanol gradient (100\%, 95\%, and 70\%). Apoptotic cells were detected using the DeadEnd Fluorometric TUNEL assay (Promega) according to the manufacturer's protocol.

NanoString gene expression analysis. Total RNA samples were analyzed using the nCounter mouse neuroinflammation (v1.0), nCounter mouse neuropathology (v1.0), and nCounter mouse myeloid (v2.0) code sets. Probe set-target RNA hybridization reactions were performed according to the manufacturer's protocol (NanoString). For each hybridization reaction, $100 \mathrm{ng}$ total RNA was used. Purified probe set-target RNA complexes from each reaction were processed and immobilized on nCounter cartridges using an nCounter Max preparation station, and transcripts were quantified on a digital analyzer (Gen, v.2). Data from each NanoString panel were first processed independently using nSolver (v.4.0) software (NanoString) as follows: following quality control checks on the individual RCC files, raw counts across samples were normalized to the mean counts for spiked synthetic DNA-positive controls present in the hybridization reactions to mitigate platform-associated sources of variation. Background thresholding was performed to the mean of negative control samples. Candidate reference genes were selected using the nCounter advanced analysis (nCAA) module (v.2.0.115), which implements the geNorm algorithm for downselection (52). Starting with a set of candidate reference genes, the algorithm identified the top 5 most stable genes for each panel. For each sample, normalization was performed by dividing the counts for each gene by the geometric mean for the 5 selected reference genes. These 3 normalized data sets were then combined in nSolver as a multi-RLF merge experiment and then input into the nCAA module for differential expression, gene set, cell type, and biological pathway analysis. The threshold for differential expression was a $\log _{2}$ fold change $>1$ and $P<0.05$.

Statistics. All analyses were performed using Prism software. Survival statistics used the log-rank test. Statistical significance of virus titers was determined using unpaired 2-tailed Student's $t$ test. A $P$ value of less than 0.05 was considered significant.

Study approval. All animal studies were conducted in compliance with the Animal Welfare Act and other federal statutes and regulations relating to animals and experiments involving animals and adheres to principles stated in the Guide for the Care and Use of Laboratory Animals, National Research Council (53). Animal experimental protocols were approved by a standing IACUC. The facilities where this research was conducted are fully accredited by the Association for Assessment and Accreditation of Laboratory Animal Care International. Animals meeting preestablished criteria were humanly euthanized after consultation with veterinary staff. Despite efforts to euthanize moribund mice, some were found dead $(n=2)$.

\section{Author contributions}

JWG and JWH designed the studies. JWG, CRC, XZ, ARG, BDC, EMM, LEW, JDS, RLB, JL, AMB, HBR, JMS, BSH, CF, and CVB performed research. JWG, CRC, and XZ analyzed data. JWG, CRC, and JWH wrote initial drafts of the manuscript. All authors contributed to the final manuscript.

\section{Acknowledgments}

Funding was provided through Defense Health Program with programmatic oversight from the Military Infectious Diseases Research Program under project 188155775. We thank the USAMRIID histology lab and the veterinary medicine division for their assistance, along with the Brian Kearney and Kathleen Gibson for providing virus through the Unified Culture Collection. We would also like to thank the Jackson Laboratory for providing early access to the K18-hACE2 mice. Opinions, interpretations, conclusions, and recommendations are ours and are not necessarily endorsed by the U.S. Army or the Department of Defense. 
Address correspondence to: Joseph W. Golden or Jay W. Hooper, Department of Molecular Virology, Virology Division, USAMRIID, 1425 Porter Street, Fort Detrick, Maryland 21702, USA. Phone: 301.6194112; Email: joseph.w.golden.ctr@mail.mil (JWG) or jay.w.hooper.civ@mail.mil (JWH).

1. Chen N, et al. Epidemiological and clinical characteristics of 99 cases of 2019 novel coronavirus pneumonia in Wuhan, China: a descriptive study. Lancet. 2020;395(10223):507-513.

2. Zhou P, et al. A pneumonia outbreak associated with a new coronavirus of probable bat origin. Nature. 2020;579(7798):270-273.

3. Yang X, et al. Clinical course and outcomes of critically ill patients with SARS-CoV-2 pneumonia in Wuhan, China: a single-centered, retrospective, observational study. Lancet Respir Med. 2020;8(5):475-481.

4. Barnes BJ, et al. Targeting potential drivers of COVID-19: neutrophil extracellular traps. J Exp Med. 2020;217(6):e20200652.

5. Salimi S, Hamlyn JM. COVID-19 and crosstalk with the hallmarks of aging. J Gerontol A Biol Sci Med Sci. 2020;null(9):glaa149.

6. Tang D, Comish P, Kang R. The hallmarks of COVID-19 disease. PLoS Pathog. 2020;16(5):e1008536.

7. Ackermann M, et al. Pulmonary vascular endothelialitis, thrombosis, and angiogenesis in Covid-19. N Engl J Med. 2020;383(2):120-128.

8. Teuwen LA, Geldhof V, Pasut A, Carmeliet P. COVID-19: the vasculature unleashed. Nat Rev Immunol. 2020;20(7):389-391

9. Connors JM, Levy JH. COVID-19 and its implications for thrombosis and anticoagulation. Blood. 2020;135(23):2033-2040.

10. Ye Q, Wang B, Mao J. The pathogenesis and treatment of the 'Cytokine Storm' in COVID-19. J Infect. 2020;80(6):607-613.

11. Vardhana SA, Wolchok JD. The many faces of the anti-COVID immune response. J Exp Med. 2020;217(6):e20200678.

12. DosSantos MF, et al. Neuromechanisms of SARS-CoV-2: a review. Front Neuroanat. 2020;14:37.

13. Hopkins C, Surda P, Whitehead E, Kumar BN. Early recovery following new onset anosmia during the COVID-19 pandemic an observational cohort study. J Otolaryngol Head Neck Surg. 2020;49(1):26.

14. Natoli S, Oliveira V, Calabresi P, Maia LF, Pisani A. Does SARS-Cov-2 invade the brain? Translational lessons from animal models. Eur J Neurol. 2020;27(9):1764-1773.

15. Li W, et al. Angiotensin-converting enzyme 2 is a functional receptor for the SARS coronavirus. Nature. 2003;426(6965):450-454.

16. Shang J, et al. Structural basis of receptor recognition by SARS-CoV-2. Nature. 2020;581(7807):221-224.

17. Hoffmann M, et al. SARS-CoV-2 cell entry depends on ACE2 and TMPRSS2 and is blocked by a clinically proven protease inhibitor. Cell. 2020;181(2):271-280.

18. Wrapp D, et al. Cryo-EM structure of the 2019-nCoV spike in the prefusion conformation. Science. 2020;367(6483):1260-1263.

19. Lan J, et al. Structure of the SARS-CoV-2 spike receptor-binding domain bound to the ACE2 receptor. Nature. 2020;581(7807):215-220.

20. Wan Y, Shang J, Graham R, Baric RS, Li F. Receptor recognition by the novel coronavirus from Wuhan: an analysis based on decade-long structural studies of SARS coronavirus. J Virol. 2020;94(7):e00127-20.

21. Gretebeck LM, Subbarao K. Animal models for SARS and MERS coronaviruses. Curr Opin Virol. 2015;13:123-129.

22. Subbarao K, et al. Prior infection and passive transfer of neutralizing antibody prevent replication of severe acute respiratory syndrome coronavirus in the respiratory tract of mice. J Virol. 2004;78(7):3572-3577.

23. Bao L, et al. The pathogenicity of SARS-CoV-2 in hACE2 transgenic mice. Nature. 2020;583(7818):830-833.

24. Glass WG, Subbarao K, Murphy B, Murphy PM. Mechanisms of host defense following severe acute respiratory syndrome-coronavirus (SARS-CoV) pulmonary infection of mice. J Immunol. 2004;173(6):4030-4039.

25. Frieman MB, et al. SARS-CoV pathogenesis is regulated by a STAT1 dependent but a type I, II and III interferon receptor independent mechanism. PLoS Pathog. 2010;6(4):e1000849.

26. Hogan RJ, et al. Resolution of primary severe acute respiratory syndrome-associated coronavirus infection requires Stat1. J Virol. 2004;78(20):11416-11421

27. Lutz C, Maher L, Lee C, Kang W. COVID-19 preclinical models: human angiotensin-converting enzyme 2 transgenic mice. Hum Genomics. 2020;14(1):20.

28. Tseng CT, et al. Severe acute respiratory syndrome coronavirus infection of mice transgenic for the human Angiotensin-converting enzyme 2 virus receptor. J Virol. 2007;81(3):1162-1173.

29. Yang XH, et al. Mice transgenic for human angiotensin-converting enzyme 2 provide a model for SARS coronavirus infection. Comp Med. 2007;57(5):450-459.

30. McCray PB, et al. Lethal infection of K18-hACE2 mice infected with severe acute respiratory syndrome coronavirus. $J$ Virol. 2007;81(2):813-821.

31. Menachery VD, et al. SARS-like WIV1-CoV poised for human emergence. Proc Natl Acad Sci U S A. 2016;113(11):3048-3053.

32. Netland J, Meyerholz DK, Moore S, Cassell M, Perlman S. Severe acute respiratory syndrome coronavirus infection causes neuronal death in the absence of encephalitis in mice transgenic for human ACE2. J Virol. 2008;82(15):7264-7275.

33. Dinnon KH, et al. A mouse-adapted model of SARS-CoV-2 to test COVID-19 countermeasures [published online August 27, 2020]. Nature. https://doi.org/10.1038/s41586.020.2708-8.

34. Sun SH, et al. A mouse model of SARS-CoV-2 infection and pathogenesis. Cell Host Microbe. 2020;28(1):124-133.e4

35. Hassan AO, et al. A SARS-CoV-2 infection model in mice demonstrates protection by neutralizing antibodies. Cell. 2020;182(3):744-753.e4.

36. Israelow B, et al. Mouse model of SARS-CoV-2 reveals inflammatory role of type I interferon signaling. J Exp Med. 2020;217(12):e20201241.

37. Yamaji-Kegan K, Su Q, Angelini DJ, Myers AC, Cheadle C, Johns RA. Hypoxia-induced mitogenic factor (HIMF/FIZZ1/ RELMalpha) increases lung inflammation and activates pulmonary microvascular endothelial cells via an IL-4-dependent mechanism. J Immunol. 2010;185(9):5539-5548.

38. Zhao Y, et al. Protection of LPS-induced murine acute lung injury by sphingosine-1-phosphate lyase suppression. Am J Respir Cell Mol Biol. 2011;45(2):426-435. 
39. Suryadevara V, et al. Sphingolipids in ventilator induced lung injury: role of sphingosine-1-phosphate lyase. Int J Mol Sci. 2018;19(1):114.

40. Blanco-Melo D, et al. Imbalanced host response to SARS-CoV-2 drives development of COVID-19. Cell. 2020;181(5):1036-1045.

41. Varga Z, et al. Endothelial cell infection and endotheliitis in COVID-19. Lancet. 2020;395(10234):1417-1418.

42. Xu X, et al. Effective treatment of severe COVID-19 patients with tocilizumab. Proc Natl Acad Sci U S A. 2020;117(20):10970-10975.

43. Park MD. Macrophages: a Trojan horse in COVID-19? Nat Rev Immunol. 2020;20(6):351.

44. Zhang W, et al. The use of anti-inflammatory drugs in the treatment of people with severe coronavirus disease 2019 (COVID19): the perspectives of clinical immunologists from China. Clin Immunol. 2020;214:108393.

45. Pence BD. Severe COVID-19 and aging: are monocytes the key? Geroscience. 2020;42(4):1051-1061.

46. Grillet F, Behr J, Calame P, Aubry S, Delabrousse E. Acute pulmonary embolism associated with COVID-19 pneumonia detectedPneumonia Detected with pulmonary CT angiography. Radiology. 2020;296(3):E186-E188.

47. Jin JM, et al. Gender differences in patients with COVID-19: focus on severity and mortality. Front Public Health. 2020;8:152

48. Li YC, Bai WZ, Hashikawa T. The neuroinvasive potential of SARS-CoV2 may play a role in the respiratory failure of COVID-19 patients. JMed Virol. 2020;92(6):552-555.

49. Hanafi R, et al. COVID-19 neurologic complication with CNS vasculitis-like pattern. AJNR Am J Neuroradiol. 2020;41(8):1384-1387.

50. Prophet EB, Mills B, Arrington JB, Sobin LH. Laboratory Methods in Histotechnology. American Registry of Pathology; 1992.

51. Liu J, Babka AM, Kearney BJ, Radoshitzky SR, Kuhn JH, Zeng X. Molecular detection of SARS-CoV-2 in formalin-fixed, paraffin-embedded specimens. JCI Insight. 2020;5(12):139042.

52. Vandesompele J, et al. Accurate normalization of real-time quantitative RT-PCR data by geometric averaging of multiple internal control genes. Genome Biol. 2002;3(7):RESEARCH0034.

53. National Research Council (US) Committee for the Update of the Guide for the Care and Use of Laboratory Animals. Guide for the Care and Use of Laboratory Animals. National Academies Press; 2011. 